\title{
Wissenslücken erkennen, die Technik sinnvoll nutzen und auf eigene Grenzen rechtzeitig reagieren
}

\section{Aus- und Weiterbildung \\ Medizinstudent veröffentlicht Lehrbuch: Wie sehen Symptome auf dunkler Haut aus?}

«Wie sieht das auf dunkler Haut aus?» Diese Frage hat der Londoner Medizinstudent Malone Mukwende schon oft gestellt, wenn es im Studium um bestimmte Symptome ging. Mit «Mind the gap» hat er nun ein Lehrbuch zum Thema veröffentlicht.

Manche Symptome sehen bei dunkelhäutigen Menschen einfach anders aus - beispielsweise Hämatome oder Ausschläge. Allerdings: In medizinischen Lehrbüchern werden in der Regel nur hellhäutige Menschen als Fallbeispiele abgebildet. Der Londononer Medizinstudent Malone Mukwende suchte lange nach einem Buch zu dem Thema. Er fand keines - also machte er sich selbst ans Werk. Denn: Auch wenn es im Medizinstudium meistens um weiße Menschen geht, hat ein Großteil der Weltbevölkerung dunkle Haut. Das Thema spiele auch eine Rolle während der aktuellen COVID19-Pandemie, erklärt der 20-Jährige. Denn wenn ein Angehöriger

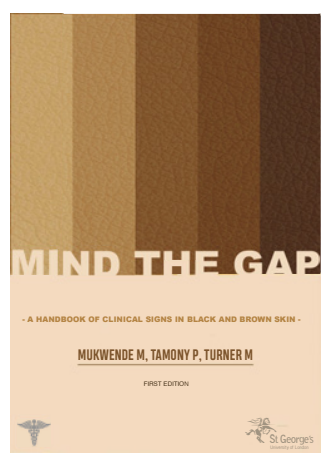
eines möglichen COVID-Patienten beispielsweise bei einer telefonischen Beratung gefragt werde, ob die Haut des Patienten blass und die Lippen bläulich seien, seien diese Fragen bei dunkelhäutigen Menschen für eine Anamnese nicht sehr hilfreich, so der Student. Die Symptome sehen bei schwarzer oder brauner Haut einfach anders aus. Das könne auch die Chance auf eine angemessene Behandlung verschlechtern.
«Das Buch ,Mind the gap' hat eine Diskussion darüber angeregt, wie stark verschiedene Patientengruppen in der medizinischen Lehre repräsentiert sind», erklärt Mukwende, «ich hoffe, dass der Inhalt des Buches und ähnlicher Stoff in Zukunft auch Bestandteil des Medizinstudiums sein werden». Mukwende, der an der University of London studiert, ist vor Kurzem in sein 3. Studienjahr gestartet. Er stammt aus Simbabwe, lebt aber seit seiner frühen Kindheit in Großbritannien. Bei seinem Projekt bekommt er auch viel Unterstützung von Seiten seiner Dozenten. So werden an seiner Universität beispielsweise Projekte gefördert, für die Studierende und Mitglieder des Lehrpersonals zusammenarbeiten.

Das Buch steht kostenlos auf der Webseite Black \& Brown Skin zum Download zur Verfügung. Dort können Ärzte und Patienten auch eigene Bildbeispiele von verschiedenen Symptomen hochladen. So will Mukwende eine große Datenbank an Beispielfotos zusammentragen und auch dafür sorgen, dass andere Mediziner dem Thema mehr Aufmerksamkeit widmen. Seit das Buch im Juni 2020 erschienen ist, ist es immerhin schon auf den Leselisten von acht britischen Medizinfakultäten gelandet.

\section{Quellen}

St. George's University of London, Black and Brown Skin: www.blackandbrownskin.co.uk

Beitrag von Stefanie Hanke. Erstveröffentlichung auf operation-karriere.de.
OPERATION
KARRIERE 


\section{Experte im Gespräch}

\section{«Es geht ja nicht darum, Ärzte zu ersetzen»}

Künstliche Intelligenz (KI) ist eines der großen Zukunftsthemen in der Medizin: Schon jetzt ist KI menschlichen Medizinern bei der Diagnose bestimmter Krankheiten überlegen. Wohin die Reise geht, erklärt im Interview der KI-Experte Prof. Dr. Martin Hirsch.

Herr Prof. Hirsch, Künstliche Intelligenz ist in der Medizin ein Thema, das die Zukunft bestimmen wird. Wie wird KI aktuell schon in der Medizin eingesetzt?

Einerseits gibt es den Bereich Machine Learning: Diese Kl ist für die Medizin unter anderem bei verschiedenen Diagnoseverfahren interessant: Sie kann beispielsweise helfen, Bilder von Hautveränderungen oder vom Augenhintergrund, aber auch Laboranalysen auszuwerten. In diesen Bereichen fallen ja große Datenmengen an - und da kann die KI nach auffälligen Werten suchen und die Ärzte so von Routineaufgaben entlasten. Dann gibt es noch einen zweiten Bereich, der aktuell immer wichtiger wird: die Entscheidungsunterstützung. Diese Art der Kl ist bei der Diagnose seltener Erkrankungen schon jetzt in jeder Ambulanz im Einsatz. Da sind die Systeme eine große Hilfe - denn seltene Erkrankungen sind zu komplex und zu vielseitig, als dass ein einziger Arzt sie überschauen könnte.

Über die Diagnosefindung haben Sie eben gesprochen - da ist KI heute schon menschlichen Ärzten überlegen. Was können Maschinen besser als Menschen?

Ein computerbasiertes KI-System liefert immer eine gleichbleibende Qualität: Es ist nie müde und oder unkonzentriert. Außerdem hat die KI viel mehr Speicherkapazität für Informationen und kann mit viel komplexeren Zusammenhängen umgehen als ein Mensch. Und die KI ist schneller. Sie kann viel mehr Optionen durchrechnen und ausprobieren. Wir Menschen müssen insgesamt cleverer sein, um viele Möglichkeiten von vornherein ausschließen zu können. Das sind die vier großen Vorteile: Komplexität, Kapazität, Geschwindigkeit und Ermüdungsfreiheit.

\section{Was können Menschen besser als Maschinen?}

Was Menschen auf absehbare Zeit einfach besser können, ist beispielsweise das Querdenken. KI erkennt vor allem Muster und vergleicht das, was sie vorgesetzt bekommt, mit bekannten Mustern. Deshalb fällt es ihr sehr schwer, Neues zu denken. Menschliches Denken ist kreativer und dadurch den Maschinen auch auf absehbare Zeit weit überlegen. Ein anderes Thema ist die Werteabwägung: Es ist nicht immer die Lösung am besten, die medizinisch am sinnvollsten ist. Jeder Mensch hat andere Vorlieben: Der eine hat nicht so ein hohes Schmerzempfinden; er erträgt lieber Schmerzen, als ein Medikament zu nehmen, bei dem er eine geistige Eintrübung in Kauf nehmen muss. Bei einem anderen Patienten ist das andersherum. Das sind keine rein sachlichen Entscheidungen - bei solchen Überlegungen kann KI noch nicht mal in Ansätzen etwas Sinnvolles beisteuern.

\section{Das klingt nicht so, als könnte die KI menschliche Ärzte auf absehbare Zeit komplett ersetzen.}

Es geht ja nicht darum, Ärzte zu ersetzen, sondern sie zu entlasten. Neben den beiden Aspekten, die wir eben besprochen haben, ist noch ein dritter Punkt wichtig, den Maschinen nie übernehmen können: Das ist die menschliche Zuwendung. Die Angst von Ärzten, durch solche Maschinen ersetzt zu werden, resultiert aus einer massiven Technisierung der Medizin in den vergangenen Jahrzehnten. Zu vielen medizinischen Apparaten kommen nun auch noch denkende medizinische Apparate hinzu. Daran sieht man, dass der menschliche Teil der Medizin etwas unter die Räder gekommen ist. Meine Hoffnung ist:Wenn der technische Teil der Medizin mehr von Maschinen übernommen wird, haben die Ärztinnen und Ärzte vielleicht wieder mehr Kapazitäten zur Verfügung, um zum menschlichen Teil der Medizin zurückzukehren.

\section{Sie haben selbst ja die App «Ada» entwickelt, die Ärzte bei der} Diagnose unterstützt. Wie reagieren die Ärzte auf so ein System?

Wer kann es Ärzten verdenken, wenn sie sich von so einer App erstmal in Frage gestellt fühlen? Wenn ein Arzt unvorbereitet mit so einem System konfrontiert wird, gerät sein Selbstverständnis mit Sicherheit erstmal ins Wanken. Aber ich glaube, dass jeder, der sich ein bisschen damit beschäftigt, sofort merkt: Das ist doch eine riesige Chance für mich! Die meisten Ärzte merken sehr schnell, dass das für sie keine wirkliche Bedrohung ist. Ich sehe eher die Gefahr, dass die Ärzte solche Systeme nicht ernstnehmen und deshalb darauf verzichten. An diesem Punkt werden wir künftig vielleicht sehen, dass Angehörige oder Patienten einen Arzt verklagen, weil er keine KI genutzt hat.

\section{Kann man sich denn vorstellen, dass Ärzte künftig verpflichtet werden, Kl zu nutzen?}

Das wird mit Sicherheit kommen. Krankenkassen werden künftig darauf bestehen, auch KI-Systeme in die Diagnose einzubeziehen, bevor sie eine sehr teure Therapie bezahlen. Heute sind ja schon Konzile üblich, bei denen mehrere erfahrene Ärzte über schwierige Fälle sprechen. Aber diese Konzile entscheiden ja nicht, sie geben nur ihre Meinung dazu ab. Das ist ja kein demokratischer Prozess. Künftig wird die KI dann wie ein zusätzlicher, technischer Kollege mit in diesen Prozess einbezogen. Eine wichtige Anforderung an $\mathrm{Kl}$ ist deshalb, dass sie auch argumentieren können muss. Sonst können die Ärzte nicht nachvollziehen, warum das System eine bestimmte Empfehlung abgibt. Am Ende entscheidet der Arzt, ob er sich dieser Argumentation anschließt oder nicht. Die Entscheidung über eine Therapie und damit auch die Verantwortung wird auch in Zukunft immer beim behandelnden Arzt bleiben.

\section{Welche Entwicklungen bei der Kl erwarten Sie für die kommenden Jahre?}

Ich denke, es wird künftig eine Art Symbiose von Arzt und künstlicher Intelligenz geben. Die KI kann dann den eher analytischen Teil übernehmen und der Arzt übersetzt das dem Patienten und berät 
inn, wenn es um die richtige Therapie geht. Außerdem wird es künftig in den Wartezimmern die Möglichkeit geben, die Anamnese mit Hilfe von Kl zu durchzuführen, beispielsweise mit einem Tablet. Der Arzt kann dann schon mit einer Vordiagnose ins Patientengespräch starten und muss nicht ganz von vorn anfangen. Das spart Zeit, in der sich die Ärztinnen und Ärzte mehr um ihre Patienten kümmern können.

\section{In 30 Jahren sind die Medizinstudierenden von heute erfahrene} Ärztinnen und Ärzte. Wagen wir mal den Blick in die Glaskugel: Wie wird die Medizin dann aussehen und welche Rolle spielt die KI? Die Gesundheitssysteme der Zukunft werden nicht mehr in den Warteräumen beginnen, sondern schon zu Hause auf dem Sofa, wenn man die Symptome in ein KI-System auf dem Smartphone eingibt, das einen dann direkt an den passenden Arzt verweist. Wenn die KI beispielsweise herausfindet, dass der Patient sehr wahrscheinlich unter einer seltenen Erkrankung leidet, dann kann er direkt zu einem Spezialisten gehen und spart sich den Weg über den Hausarzt. Denn der kennt diese Krankheit vermutlich nicht. Diese Art von «Care Navigation» wird Ärzten und Patienten Zeit sparen und auch die Kosten im Gesundheitssystem senken.

Die andere große Umstellung wird ein Wechsel von einem krankheitsbasierten zu einem gesundheitsbasierten System sein. Heute gehen die Menschen zum Arzt, wenn sie krank sind und wieder gesund werden wollen. In 30 Jahren werden wir viel stärker fragen, welche Veranlagungen zu einem gesundheitlichen Risiko werden könnten. Das sind beispielsweise genetische Dispositionen, der Lebensstil, die psychische Verfassung oder die Familiengeschichte. Jeder Mensch hat bestimmte Schwachstellen - und ein KI-System auf dem Smartphone wird diese Schwachstellen kennen und gezielt nach Biomarkern suchen, die darauf hinweisen, dass sich eine Krankheit anbahnt. Das kann ein bestimmter Blutwert sein, der über Jahre leicht ansteigt. Der Wert ist zwar noch im grünen Bereich, aber die Tendenz weist darauf hin, dass hier ein Problem entstehen könnte. Wenn ich das weiß, bin ich eher motiviert, etwas an meinem Lebensstil zu ändern: also mich mehr zu bewegen oder auf Alkohol zu verzichten - lange, bevor das erste Symptom entsteht. Ich glaube, dass die KI-Systeme uns in ein Zeitalter der personalisierten Prävention führen werden.

\section{Welche Rolle wird KI künftig im Medizinstudium spielen?}

Wichtig ist, dass die Studierenden künftig im Studium lernen, wie sie mit der Kl arbeiten können - das muss ein Teil der Ausbildung sein, damit sie als Ärztinnen und Ärzte später urteilsfähig bleiben. Denn später werden sie immer mit KI-Systemen zusammenarbeiten - vor allem, wenn die Fälle komplexer sind. Die KI wird ihnen assistieren - und sie müssen ja lernen, die Ratschläge der KI mit einer kritischen Distanz zu beurteilen, ohne ihr ärztliches Denken an die Maschinen zu delegieren. Bei Rechenaufgaben greift man ja heute lieber zum Taschenrechner und überprüft das Ergebnis nicht mehr im Kopf. Das sollten wir in der Medizin aber vermeiden. Denn ein KI-System ist immer ein technisches System und wird mit Fehlern behaftet sein.

\section{Womit fühlen Sie sich denn als Patient besser: Mit einem mensch-} lichen Arzt oder mit einem KI-System?

Am wohlsten fühle ich mich mit einem menschlichen Arzt, der von einer Kl unterstützt wird. Das wird auch die Zukunft sein.

Beitrag von Stefanie Hanke. Erstveröffentlichung auf Ärztestellen.de

\section{ÄRZTESTELLEN DER STELLENMARKT DES DEUTSCHEN ÄRZTEBLATTES}

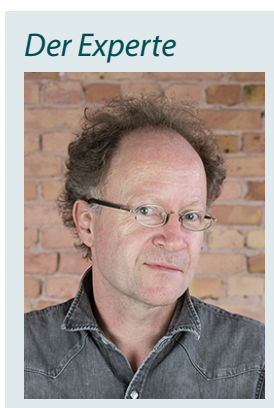

Prof. Dr. Martin Hirsch ist Humanbiologe und promovierter Neurowissenschaftler. Er gründete 2010 das Gesundheits- und Technologieunternehmen Ada Health und entwickelte die App «Ada», die eine wissenschaftlich basierte Symptomanalyse mit Hilfe von KI durchführt und damit Ärzten und Patienten bei der Diagnosestellung hilft. Zum 1. Januar 2020 wurde Martin Hirsch auf die neu eingerichtete Professur «Künstliche Intelligenz in der Medizin» an der Philipps-Universität Marburg berufen. Bild: () Ada Health GmbH 2020

\section{Aus- und Weiterbildung}

\section{«Kündigen oder nicht? Wann ist Zeit zu gehen?»}

Ärger mit dem Chef, den ungeliebten Kollegen und überhaupt... manchmal denkt man «nur raus hier» und würde am liebsten sofort die Flinte ins Korn werfen. Klar, kündigen geht ganz einfach. Der Brief ist schnell geschrieben. Aber ist das immer klug? Spontan sicherlich nicht. Schlechte Tage gibt es überall und eine so weitreichende Handlung will - auch bei bester Arbeitsmarktsituation - wohl überlegt sein.

«Abgesehen vom ,normalem' Alltagsfrust gibt es, meiner Erfahrung nach, einen entscheidenden Hauptaspekt für einen Arbeitsplatzwechsel. Und das ist die Frage: Kann ich in meinem derzeitigen Job meine inneren Werte leben oder muss ich ständig dagegen arbei- ten?», sagt Silke Wüstholz, Coachin für Ärzte und Pflegepersonal. Und in diesen inneren Werten steckt ziemlich viel drin: keine oder zu wenig Zeit für die Patienten, schlechte interdisziplinäre Beziehungen, unverständliche Entscheidungen im Management und immer öfter mangelnde Work-Life-Balance. «Denn auch in punkto Arbeitszeiten geht es darum, ob mir dafür so viel Lebenszeit nicht zu schade ist. Vielleicht möchte ich mehr Zeit für meine Familie oder mein Hobby haben?», fügt Wüstholz an. Schließlich verbringt schon der Durchschnittsdeutsche ein gutes Drittel seines Lebens im Beruf ein Arzt oft mehr. Und wie zufrieden man damit ist, strahlt weit ins Privatleben hinein und prägt sogar die eigene Identität. 


\section{Die Bestandsaufnahme: Wo stehe ich?}

Als erstes empfiehlt sich eine Standortbestimmung: Wo stehe ich? Was ist mir wichtig? Was fehlt mir? «Eine gute Methode ist, jeden Tag oder wenigstens jeden zweiten kurz aufzuschreiben, was mir heute gut gefallen hat und was nicht so gut lief», rät die Expertin. Das hilft, die Gedanken zu sortieren, Gedankenschleifen zu vermeiden und ergibt direkt eine klarere Sicht.

Der nächste Schritt ist ein baldiges Vorsprechen beim Chef. Denn im besten Fall kann der Ärger sofort ausgeräumt werden. «Manchmal sind es nur Missverständnisse, dass etwas nicht gut kommuniziert wurde», so Wüstholz und hat einen ganz praktischen Tipp parat: «Nehmen Sie eine konkrete Idee mit ins Gespräch, was die Führungskraft tun könnte, um Ihr Anliegen zu verbessern. » Bei dieser Gelegenheit sieht man dann auch, ob der Vorgesetzte generell Verständnis für die Nöte seiner Mitarbeiter aufbringt oder alles wegbügelt - dann wird man auch künftig wenig erreichen. Wer die Stelle erst vor kurzem angetreten hat, kann auch an das Einstellungsgespräch erinnern und sagen: «lch hatte den Eindruck, dass mir das hier ermöglicht wird...».

Zudem sollte man gleich bei diesem Termin fragen: «Sind Sie damit einverstanden, wenn ich Sie in zwei Monaten noch einmal darauf anspreche?» Dann ist der Weg dafür bereits geebnet und man hat sich auch selbst dazu verpflichtet. Bis dahin ist dann allerdings etwas Geduld angebracht, denn: «Viele Menschen denken, ich hab' das doch jetzt gesagt und da muss doch auch was passieren. Aber man darf nicht vergessen, dass das Gegenüber noch 1.000 andere Dinge klären muss. Sie sind also selbst in der Pflicht noch mal nachzuhaken», erläutert die 50-jährige Beraterin und ergänzt: «Alles natürlich ganz freundlich, diplomatisch und mit klarer Haltung.»

\section{Die Selbstanalyse: Welche Signale sende ich?}

Zwischenzeitlich ist ein Blick auf das eigene Auftreten empfehlenswert. «Manche meinen, der Chef muss doch sehen, dass ich beispielsweise mehr Verantwortung übernehmen will, aber sie verhalten sich total zurückhaltend. Dann liegt meist ein anderes Problem darunter», schildert Wüstholz. So hat sie es schon oft erlebt, dass ihre Klienten mit dem Gedanken «das ist ja alles überhaupt nicht mehr auszuhalten» in die Beratung gekommen sind. «Doch in Wirklichkeit haben sie sich selbst klein gemacht aufgrund alter Ängste oder Kindheits-Erfahrungen, nach dem Motto ,lieber wegducken'. Also wird das Vorpreschen erst gar nicht probiert. Stattdessen steigern sie sich in die vermeintliche Ablehnung hinein und denken: ,ich kann hier ja sowieso nichts ändern'», erklärt die frühere Fachkrankenschwester für Anästhesie- und Intensivmedizin.

Das betrifft übrigens beide Geschlechter. In diesem Fall geht es darum, die Haltung zu ändern und die Kommunikation zu verbessern: «Nicht wenige Ärztinnen, aber auch Ärzte, müssen erst lernen, ihre eigenen Interessen deutlich zu vertreten. Hilfreich dafür ist zum Beispiel, entsprechende Sätze und Tonlagen einzuüben und immer wieder in Anwesenheit des Chefs zu sagen: ,Oh, das will ich auch gern machen.' So kann man sich einfach kontinuierlich platzieren.»

\section{Vor dem Neustart: Preisfrage stellen}

Liegt es aber nicht an einem selbst und bringen auch Gespräche mit Vorgesetzten nichts, rückt die Kündigung selbstverständlich näher

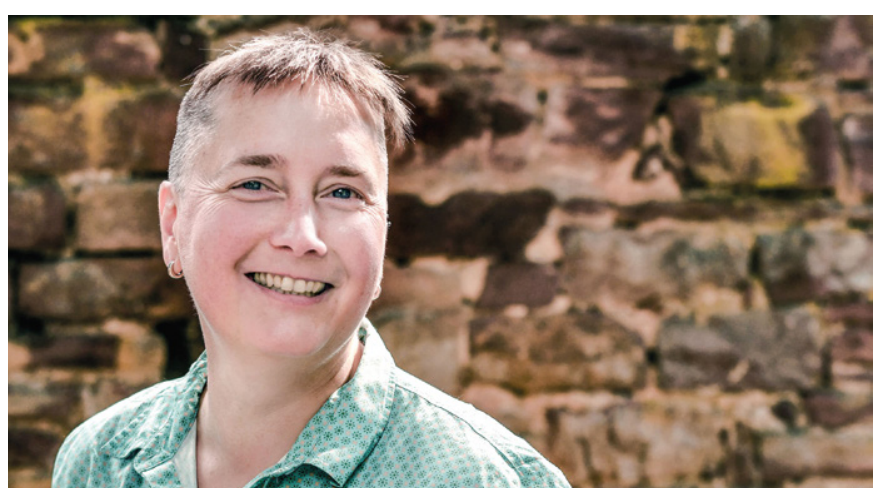

Silke Wüstholz coacht Ärzte und Pflegepersonal. Bild: @ Elisa Reznicek

Zeit, die Preisfragen zu stellen: Was kostet es mich, wenn ich bleibe? Was kostet es mich, wenn ich gehe? Wer bezahlt welchen Preis? Was kostet es die Menschen, mit denen ich zu tun habe? «Es herrscht zwar Ärztemangel, aber vielleicht bin ich durch meine Familie örtlich gebunden. Dann haben meine Forderungen natürlich weniger Wumms», betont Wüstholz.

Dennoch: Wird es zum Dauerzustand, dass ich mich zur Arbeit schleppe, keine Freude mehr habe und nicht mehr zur Ruhe komme, auch weil die Arbeitsbelastung langfristig schlichtweg zu hoch ist, hilft nur noch ein Jobwechsel, bevor die eigene Gesundheit in Gefahr gerät. «Viele denken, ich muss mir beweisen, dass ich das hier schaffe, und warten deshalb zu lange. Ich habe nicht wenige Ärzte beraten, die waren entweder kurz vor dem Burn-Out oder schon tief drin», erzählt die Karlsruherin. Leider sei es gerade für Mediziner nach wie vor ein ganz großer Schritt, sich professionelle Unterstützung zu holen. Denn das habe immer noch ein Geschmäckle, insbesondere für Männer. Und wer kommt, meint: «Eins sag ich dir gleich: Ich mach alles, nur keine Rollenspiele», berichtet die Fachfrau nun schmunzelnd. Die gibt's bei ihr aber eh nicht, stattdessen setzt sie zum Beispiel auf provokative Settings: «Wenn ich sehr kognitive Menschen berate, ist es sinnvoll, das Problem zu überzeichnen», erklärt sie und beschreibt ein Beispiel: «Hat jemand Angst, vor einer Gruppe zu reden, hilft die Vorstellung, da sitzen lauter Krokodile, die warten nur darauf dich aufzufressen. Und dann wird meist gelacht. Das hört sich vielleicht banal an, zeigt aber, wie surreal die eigenen Barrieren sind, die man sich im Kopf so zurechtbastelt." Im Sinne der bekannten Karikatur, eine Katze schaut in den Spiegel und ein Löwe schaut zurück... Gerade Ärzte würden von außen extrem souverän wirken, als könnte sie nichts erschüttern, aber tatsächlich hätten sie oft große Ängste - auch davor, Fehler zu machen, was sie aber ständig überspielen.

Egal, ob man in seinem Job bleibt oder geht: Entscheidend sind am Ende übrigens fast nie die fachlichen Dinge, sondern die Emotionen, wie man sich dabei fühlt. «Deshalb sollte man generell ab und zu mal bewusst reflektieren, ob man noch am richtigen Platz ist, mit einem Coach oder einem guten Freund, so wie man auch regelmäßig zum Zahnarzt geht», resümiert die klinikerfahrene Coachin.

Beitrag von Gerti Keller. Erstveröffentlichung auf Operation Karriere.de KARRIERE
80

Kompass Autoimmun 2021;3:77-80

DOI: $10.1159 / 000516586$ 